\title{
QUEEN'S
UNIVERSITY
BELFAST
}

\section{Testing the Spectroscopic Extraction of Suppression of Convective Blueshift}

Miklos, M., Milbourne, T. W., Haywood, R. D., Phillips, D. F., Saar, S. H., Meunier, N., Cegla, H. M., Dumusque, X., Langellier, N., Maldonado, J., Malavolta, L., Mortier, A., Thompson, S., Watson, C. A., Cecconi, M., Cosentino, R., Ghedina, A., Li, C-H., López-Morales, M., ... Walsworth, R. L. (2020). Testing the Spectroscopic Extraction of Suppression of Convective Blueshift. The Astrophysical Journal, 888(2), 117. https://doi.org/10.3847/1538-4357/ab59d5

\section{Published in:}

The Astrophysical Journal

\section{Document Version:}

Publisher's PDF, also known as Version of record

\section{Queen's University Belfast - Research Portal:}

Link to publication record in Queen's University Belfast Research Portal

\section{Publisher rights}

Copyright 2020 American Astronomical Society. This work is made available online in accordance with the publisher's policies. Please refer to any applicable terms of use of the publisher.

\section{General rights}

Copyright for the publications made accessible via the Queen's University Belfast Research Portal is retained by the author(s) and / or other copyright owners and it is a condition of accessing these publications that users recognise and abide by the legal requirements associated with these rights.

Take down policy

The Research Portal is Queen's institutional repository that provides access to Queen's research output. Every effort has been made to ensure that content in the Research Portal does not infringe any person's rights, or applicable UK laws. If you discover content in the Research Portal that you believe breaches copyright or violates any law, please contact openaccess@qub.ac.uk. 


\title{
Testing the Spectroscopic Extraction of Suppression of Convective Blueshift
}

\author{
M. Miklos ${ }^{1,2}$, T. W. Milbourne ${ }^{1,2}$ (1), R. D. Haywood ${ }^{2,14}$ (1) D. F. Phillips ${ }^{2}$, S. H. Saar ${ }^{2}$, N. Meunier ${ }^{3}$, H. M. Cegla ${ }^{4,15}$ (1),

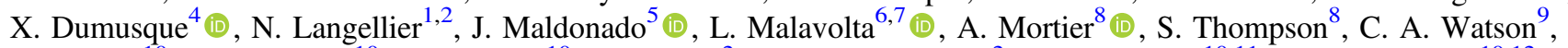

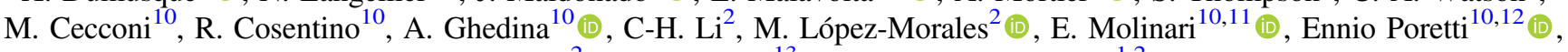 \\ D. Sasselov ${ }^{2}$ (D) A. Sozzetti ${ }^{13}$ (D), and R. L. Walsworth ${ }^{1,2}$ \\ ${ }^{1}$ Department of Physics, Harvard University, 17 Oxford Street, Cambridge MA 02138, USA \\ ${ }^{2}$ Harvard-Smithsonian Center for Astrophysics, Cambridge, MA 02138, USA \\ ${ }^{3}$ Univ. Grenoble Alpes, CNRS, IPAG, F-38000 Grenoble, France \\ ${ }^{4}$ Observatoire de Genève, Université de Genève, 51 chemin des Maillettes, 1290 Versoix, Switzerland \\ ${ }_{6}^{5}$ INAF-Osservatorio Astronomico di Palermo, Piazza del Parlamento 1, I-90134 Palermo, Italy \\ ${ }^{6}$ INAF-Osservatorio Astronomico di Padova, Vicolo dell'Osservatorio 5, I-35122 Padova, Italy \\ ${ }^{7}$ Dipartimento di Fisica e Astronomia "Galileo Galilei," Università di Padova, Vicolo dell‘Osservatorio 3, I-35122 Padova, Italy \\ ${ }_{9}$ Astrophysics Group, Cavendish Laboratory, University of Cambridge, J.J. Thomson Avenue, Cambridge CB OHE, UK \\ 9 Astrophysics Research Centre, School of Mathematics and Physics, Queen's University Belfast, Belfast BT7 1NN, UK \\ ${ }^{10}$ INAF-Fundacion Galileo Galilei, Rambla Jose Ana Fernandez Perez 7, E-38712 Brena Baja, Spain \\ ${ }^{11}$ INAF-Osservatorio Astronomico di Cagliari, Via della Scienza 5, I-09047 Selargius CA, Italy \\ ${ }_{12}^{12}$ INAF-Osservatorio Astronomico di Brera, Via E. Bianchi 46, I-23807 Merate (LC), Italy \\ ${ }^{13}$ INAF-Osservatorio Astrofisico di Torino, via Osservatorio 20, I-10025 Pino Torinese, Italy \\ Received 2019 October 22; revised 2019 November 18; accepted 2019 November 19; published 2020 January 15
}

\begin{abstract}
Efforts to detect low-mass exoplanets using stellar radial velocities (RVs) are currently limited by magnetic photospheric activity. Suppression of convective blueshift is the dominant magnetic contribution to RV variability in low-activity Sun-like stars. Due to convective plasma motion, the magnitude of RV contributions from the suppression of convective blueshift is related to the depth of formation of photospheric spectral lines for a given species used to compute the RV time series. Meunier et al. used this relation to demonstrate a method for spectroscopic extraction of the suppression of convective blueshift in order to isolate RV contributions, including planetary RVs, that contribute equally to the time series for each spectral line. Here, we extract disk-integrated solar RVs from observations over a $2.5 \mathrm{yr}$ time span made with the solar telescope integrated with the HARPS-N spectrograph at the Telescopio Nazionale Galileo (La Palma, Canary Islands, Spain). We apply the methods outlined by Meunier et al. We are not, however, able to isolate physically meaningful contributions due to the suppression of convective blueshift from this solar data set, potentially because our data set is taken during solar minimum when the suppression of convective blueshift may not sufficiently dominate activity contributions to RVs. This result indicates that, for low-activity Sun-like stars, one must include additional RV contributions from activity sources not considered in the Meunier et al. model at different timescales, as well as instrumental variation, in order to reach the submeter per second RV sensitivity necessary to detect low-mass planets in orbit around Sunlike stars.
\end{abstract}

Unified Astronomy Thesaurus concepts: Radial velocity (1332); Solar activity (1475); Solar faculae (1494); Plages (1240); Solar granulation (1498); Sunspots (1653); Exoplanet detection methods (489)

\section{Introduction}

The radial velocity (RV) method is the principal technique for constraining the masses of exoplanets (Mayor \& Queloz 1995). It provides complementary information to the transit method, e.g., as used by the Kepler and TESS spacecraft (Borucki et al. 2010; Howell et al. 2014; Ricker et al. 2014) and ground-based transit surveys. The Keplerian reflex motion induced in a Sun-like star by an Earth-mass planet in the habitable zone is of order $10 \mathrm{~cm} \mathrm{~s}^{-1}$ (Fischer et al. 2016), the target sensitivity of next-generation spectrographs (Pepe et al. 2010). However, contributions to observed stellar RVs from photospheric stellar activity often exceed $1 \mathrm{~m} \mathrm{~s}^{-1}$ even in the quietest Sun-like stars, posing a significant barrier to the detection of exoplanets by the RV method (e.g., Saar \& Donahue 1997; Schrijver \& Zwaan 2000; Isaacson \& Fischer 2010; Motalebi et al. 2015). Several recent works

\footnotetext{
14 NASA Sagan Fellow.

15 CHEOPS Fellow, SNSF NCCR-PlanetS.
}

describe a variety of models to mitigate the effects of magnetic activity on stellar RVs. One approach has been to study the Sun as a star, extracting solar activity estimates from images of the solar surface (Meunier et al. 2010a; Haywood et al. 2016; Milbourne et al. 2019) and comparing to simultaneous diskintegrated spectral measurements. In order to reduce unwanted stellar signals from exoplanet searches, however, methods for extracting stellar activity directly from spectra, and not from ancillary data sets, must be developed.

For Sun-like stars with low activity, suppression of convective blueshift due to photospheric plage (hereafter $\mathrm{RV}_{\text {conv }}$ ) dominates over the wavelength-independent photometric effects due to spots, or RV shifts induced by Earth-like exoplanets (Meunier et al. 2010b; Dumusque et al. 2014; Haywood et al. 2016; hereafter $\mathrm{RV}_{\text {sppl }}$ ). Meunier et al. (2017a; hereafter M17) have developed one model to isolate $\mathrm{RV}_{\text {conv }}$ contributions based on the observed nonlinear relationship between relative depths and absolute RV blueshifts of spectral lines of a given species (here neutral iron) driven by plasma flow in granules, as described in Gray (2009), Reiners et al. 
(2016), Meunier et al. (2017b), and Gray \& Oostra (2018). The exact physical origin of this observed correlation is non-trivial: a correct description of spectral line formation necessitates the summation of many different line profiles, each formed at different depths in the photosphere, and requires a full threedimensional treatment (e.g., see Nordlund et al. 2009; Stein 2012; Cegla et al. 2013; Bergemann et al. 2019 and references therein). An intuitive (though inexact) understanding of this relationship may be determined by considering a simplified 1D picture: in this model, rising plasma low in the photosphere exhibits strong RV blueshift, while plasma closer to the surface has most of its motion directed tangentially as it merges into intergranular lanes, thus exhibiting less RV blueshift (Dravins et al. 1981). While many factors such as temperature, electron pressure, and atomic constants affect spectral line relative depth (Gray 2005), for spectral lines of a given atomic species, line depth shows strong anti-correlation with height of formation in the stellar photosphere. Therefore, the absolute RV blueshift shows a strong, nonlinear relationship with line depth, commonly referred to as the third signature of stellar granulation (Gray 2009). M17 leverage the dominance of $\mathrm{RV}_{\text {conv }}$ to write the $\mathrm{RV}$ time series derived from a set of lines $s_{0}$ as

$$
\mathrm{RV}_{0}=\mathrm{RV}_{\mathrm{sppl}}+\mathrm{RV}_{\text {conv }}
$$

where $\mathrm{RV}_{0}$ is the $\mathrm{RV}$ measured with this specific line list. $R V_{\text {conv }}$ are line list-dependent contributions due to the suppression of convective blueshift, and $\mathrm{RV}_{\mathrm{sppl}}$ are photometric variations (e.g., spots and plage), planetary signals, or other RV sources that are the same for all spectral lines.

M17 makes use of the nonlinear relationship between line depth and convective shift by writing an RV time series from a sublist $s_{1}$ of $s_{0}$ with a restricted flux range as

$$
\mathrm{RV}_{1}=\mathrm{RV}_{\text {sppl }}+\alpha \mathrm{RV}_{\text {conv }}
$$

where $\alpha$ is the ratio of the mean shift in RV due to suppression of convective blueshift for line list $s_{1}$ compared to line list $s_{0}$. Based on the third signature of granulation (Gray 2009), we would expect $\alpha<1$ for a sublist $s_{1}$ comprised of strong lines formed close to the top of the photosphere, and $\alpha>1$ for a sublist of weak lines, formed deep in the photosphere. If a precise value for $\alpha$ is known or can be inferred, we can invert the observed $\mathrm{RV}_{0}$ and $R V_{1}$ time series to extract time series of interest $\mathrm{RV}_{\text {conv }}$ and $\mathrm{RV}_{\text {sppl }}$.

Using time series $\mathrm{RV}_{\text {conv }}$ and $\mathrm{RV}_{\text {sppl }}$ extracted from solar photospheric images, M17 constructed synthetic time series $\mathrm{RV}_{0}$ and $\mathrm{RV}_{1}$ using a value for $\alpha$ fitted from a solar atlas (Kurucz et al. 1984) and added white noise. The authors then test several methods for estimating $\alpha$ on these synthetic time series, finding good convergence for the value of $\alpha$ across the methods (within $5 \%$ of the true value for low-noise conditions; M17). Using this calculated value of $\alpha$, they then recover and validate the original $\mathrm{RV}_{\text {sppl }}$ time series. Ideally, this technique could be utilized to correct $\mathrm{RV}$ time series for $\mathrm{RV}_{\text {conv }}$ contributions to lower the RV activity threshold.

On real data, determining an absolute scale for radial velocities is challenging, making it difficult to precisely determine $\alpha$. M17 apply these methods to HARPS exposures of HD 207129 but find no agreement for values of $\alpha$ derived by different estimation methods, which they attribute to infrequent observations and low signal-to-noise ratios (S/Ns).
Using the solar telescope (Phillips et al. 2016) and the HARPS-N spectrograph at the Telescopio Nazionale Galileo (TNG; Cosentino et al. 2012), we extract high-resolution diskintegrated solar spectra (Dumusque et al. 2015). We now have more than 50,000 high-SNR solar exposures spanning over $4 \mathrm{yr}$ of observing (Collier Cameron et al. 2019). In this work, we apply Meunier's methods to the first $2.5 \mathrm{yr}$ of the solar data set (from Summer 2015-2017 November) attempt to recover a precise value of $\alpha$ for use in reconstructing $\mathrm{RV}_{\text {conv }}$ and $\mathrm{RV}_{\text {sppl }}$. In Section 2, we discuss our method for extracting line-by-line RVs from the HARPS-N solar spectra. In Section 3, we discuss various techniques for determining $\alpha$ from the resulting $\mathrm{RV}$ time series, and attempt to compute consistent $\alpha$ values. We conclude in Section 4 with a discussion of the resulting values, and possible explanations for why the model does not reduce RV rms on our data set.

\section{Methods}

\subsection{Extracting RVs from HARPS-N Spectra}

Third signature plots in the literature are often based on neutral iron lines to demonstrate the relationship between relative depth and absolute convective blueshift (Gray 2009; Reiners et al. 2016; Gray \& Oostra 2018). In order to compare lines known to exhibit the third signature effect, we consider a line list from the NIST database for Fe I lines. ${ }^{16}$ We extract disk-integrated HARPS-N solar spectra over a $2.5 \mathrm{yr}$ span, with an average of 51 exposures per day. We cut data taken in overcast weather, as identified using the HARPS-N exposure meter, and reject data for any day with five or fewer exposures. Each spectrum is shifted to a heliocentric reference frame using relative velocities from the JPL Horizons ephemeris (Giorgini et al. 1996). We normalize the spectrum continuum by dividing by the corresponding blaze measurement-we propagate the photon shot noise error from each into the fits of each spectral line, as described below.

For each individual spectral line, we stack the Dopplershifted measurements from a given day to produce a composite line profile for each day. This approach, stacking data from different exposures instead of averaging over multiple exposures, avoids interpolating data onto a common wavelength grid. We fit the line core $(0.2 \AA$ total $)$ with Gaussian profiles to extract relative depth, and line center $(0.1 \AA$ total $)$ with second-degree polynomials to measure the RV. ${ }^{17} \mathrm{We}$ adopted polynomial fits to best mirror the methods of M17. We then convert these line-center positions from wavelength to $\mathrm{RV}$. This process is illustrated in Figure 1. Removing poorly fit and blended lines results in a final list of 765 spectral lines, as seen in Table 1.

The relative velocities derived reproduce the shape of the third signature curve from Reiners et al. (2016) up to an overall offset, as shown in Figure 2. This offset may result from differences between the line lists or instruments used.

\subsection{Zeroing RV Time Series}

It is challenging to extract absolute RVs from spectral data. In accounting for blueshifts of individual spectral lines, we must identify the hypothetical RV value achieved in the

\footnotetext{
16 https://physics.nist.gov/PhysRefData/ASD/lines_form.html

17 Full lists of each Gaussian fit parameter as a function of time for each spectral line are available online at doi:10.5281/zenodo.3541149.
} 

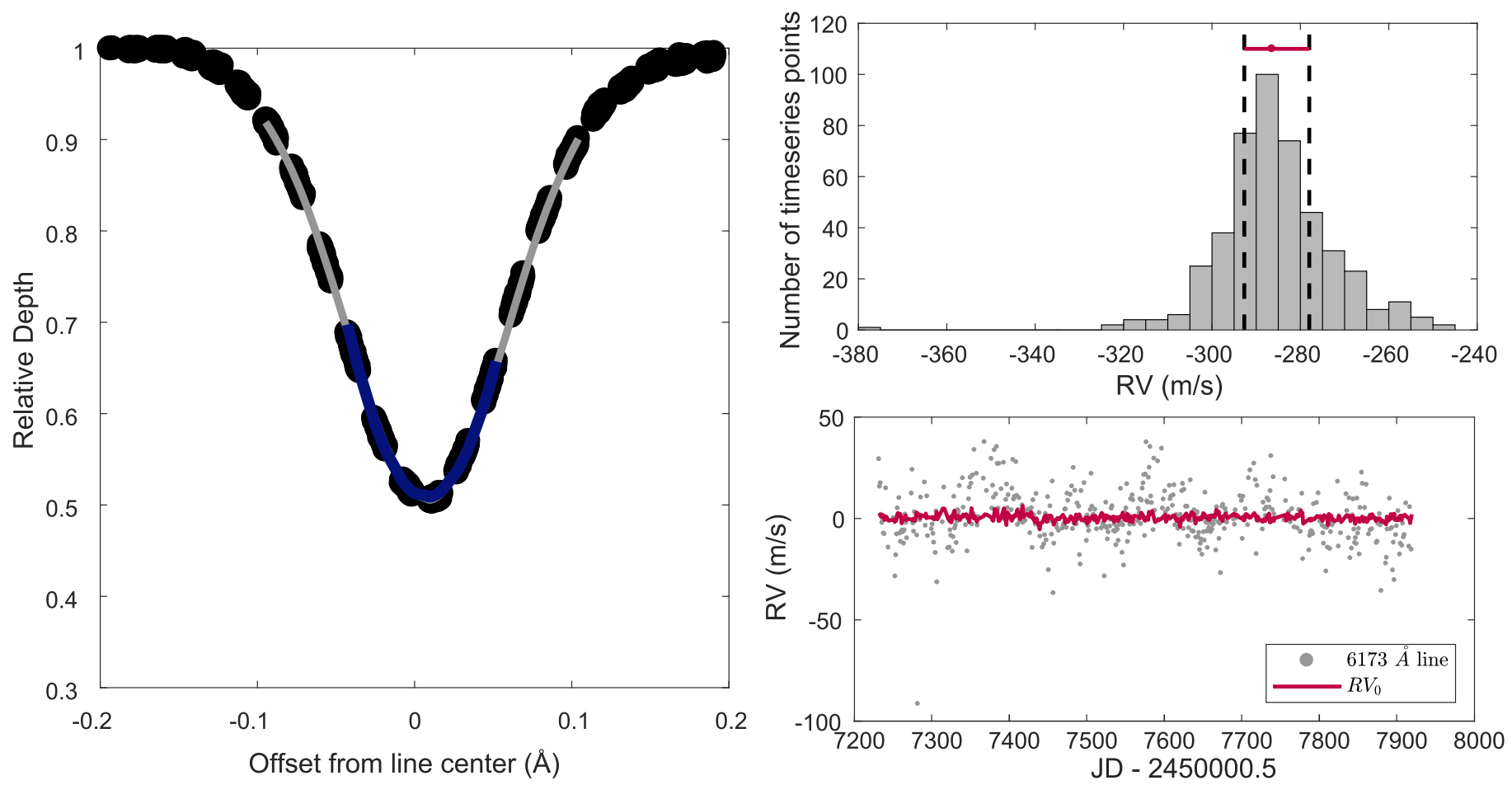

Figure 1. Left: illustration of boundaries of observed points (black) included in fits for Gaussian (gray curve) and polynomial (dark blue curve) fits for a representative line (at $6173 \AA$ ). Top right: demonstration of the zeroing procedure for the same $6173 \AA$ line-the average of the middle two quartiles of RV values per line is subtracted off. Bottom right: zeroed RV time series for $6173 \AA$ line (gray), compared to average time series for all lines, $\mathrm{RV}_{0}$ (red).

Table 1

First 10 Iron Lines Used in This Analysis

\begin{tabular}{l}
\hline \hline Wavelength $(\AA)$ \\
\hline 3922.91 \\
3946.99 \\
3948.10 \\
3975.21 \\
3995.98 \\
4000.25 \\
4000.46 \\
4001.66 \\
4022.74 \\
4047.30 \\
$\vdots$
\end{tabular}

Note. An extended line list containing all 765 spectral lines used in our analysis is available online at doi:10.5281/zenodo.3541149.

absence of stellar activity, which will vary from line to line, in a manner that is robust against outlier points or noise. We zero the RV time series per spectral line to account for this absolute blueshift. We sort time series observations by RV value per line, and subtract the average of the middle two quartiles, as shown in Figure 1. In selecting this range, we assume that lowactivity days will fall close to the median value; by subtracting the average value for the low-activity days, we aim to identify the hypothetical no-activity point for each line while avoiding bias in our zero-point due to outliers.

\subsection{Finding RVs from Sublists}

Following the procedure of M17, we identify line sublists by relative depth. We take variance-weighted means of the entire line list $\left(s_{0}\right)$, lines with relative depth $.5-.95\left(s_{1}\right)$, and lines with

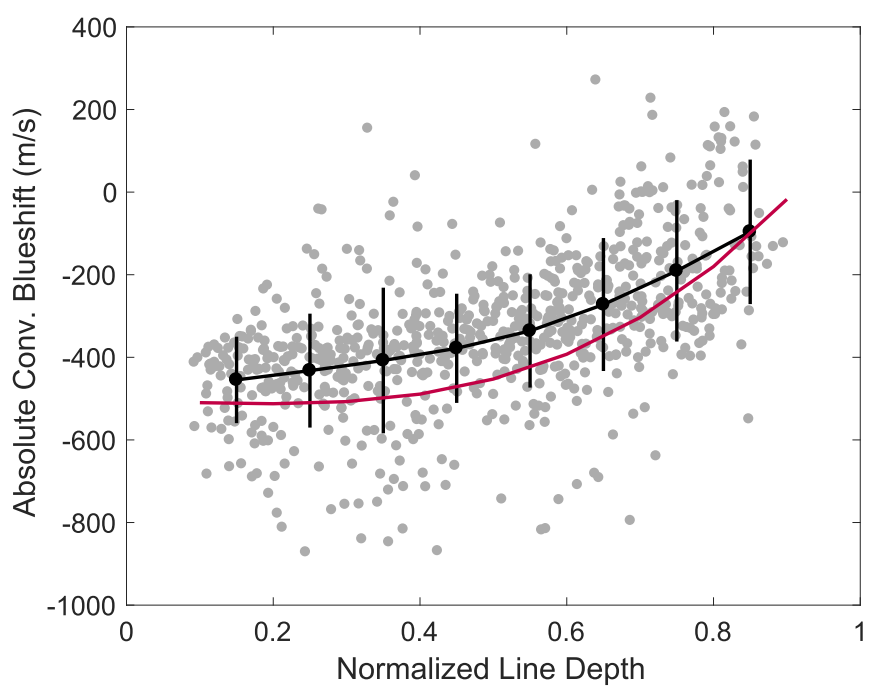

Figure 2. Third signature of stellar granulation trend demonstrated in Fe I list extracted from NIST database. Lines are binned in 0.1 relative depth bins: black dots show the average value per bin, and error bars show standard deviation per bin. The red curve shows the polynomial of best fit from Reiners et al. (2016).

relative depth .05-.5 $\left(s_{2}\right)$, to extract $\mathrm{RV}_{0}, \mathrm{RV}_{1}$, and $\mathrm{RV}_{2}$, respectively. The $\mathrm{RV}$ errors are computed from fit error bars on the line-center parameter, which incorporate propagated shot noise from the raw spectra. Features of these time series are listed in Table 2, while the time series themselves are given in Table 3.

To validate our extracted time series, we compare to the HARPS-N Data Reduction System (DRS) RVs (Baranne et al. 1996; Sosnowska et al. 2012). Figure 3 shows a Lomb-Scargle periodogram comparison of the two time series (Zechmeister \& 
Table 2

Features of RV Time Series Extracted from HARPS-N/Solar Telescope Daily Binned Spectra

\begin{tabular}{lrrr}
\hline \hline & $\mathrm{RV}_{0}$ & $\mathrm{RV}_{1}$ & $\mathrm{RV}_{2}$ \\
\hline Relative Depth & $.05-.95$ & $.5-.95$ & $.05-.5$ \\
Number of Lines & 765 & 386 & 379 \\
Standard Deviation $\left(\mathrm{m} \mathrm{s}^{-1}\right)$ & 1.50 & 1.64 & 1.74 \\
Mean $\left(\mathrm{m} \mathrm{s}^{-1}\right)$ & .47 & .37 & .59 \\
\hline
\end{tabular}

Table 3

The Extracted Time Series $\mathrm{RV}_{0}, \mathrm{RV}_{1}$, and $\mathrm{RV}_{2}$ Used in This Analysis

\begin{tabular}{lcccc}
\hline \hline $\begin{array}{l}\mathrm{JD}- \\
2450000.5\end{array}$ & $\begin{array}{c}\mathrm{RV}_{0} \\
\left(\mathrm{~m} \mathrm{~s}^{-1}\right)\end{array}$ & $\begin{array}{c}\mathrm{RV}_{1} \\
\left(\mathrm{~m} \mathrm{~s}^{-1}\right)\end{array}$ & $\begin{array}{c}\mathrm{RV}_{2} \\
\left(\mathrm{~m} \mathrm{~s}^{-1}\right)\end{array}$ & $\begin{array}{c}\mathrm{RV}_{\text {drs }} \\
\left(\mathrm{m} \mathrm{s}^{-1}\right)\end{array}$ \\
\hline 7232.51 & 1.34 & 1.26 & 1.46 & 5.66 \\
7233.54 & 2.00 & 2.15 & 1.81 & 6.71 \\
7234.51 & 0.96 & -0.16 & 2.38 & 6.24 \\
7235.49 & 0.99 & 0.06 & 2.15 & 6.89 \\
7236.51 & 1.13 & -0.34 & 2.99 & 7.48 \\
7237.49 & 1.79 & 0.68 & 3.19 & 7.12 \\
7238.56 & 0.59 & -0.32 & 1.75 & 5.25 \\
7239.43 & -0.70 & -1.46 & 0.25 & 5.38 \\
7241.55 & 1.00 & 0.07 & 2.18 & 5.34 \\
7244.47 & -1.08 & -2.58 & 0.82 & 2.31 \\
$\vdots$ & $\vdots$ & $\vdots$ & $\vdots$ & $\vdots$ \\
\hline
\end{tabular}

Note. The RVs derived from the HARPS-N DRS $\left(\mathrm{RV}_{\mathrm{DRS}}\right)$ are also provided as a point of comparison. The first $10 \mathrm{RV}$ values are given here-an extended list containing all values is available at doi:10.5281/zenodo.3541149.

Kürster 2009; VanderPlas et al. 2012; VanderPlas \& Ivezić 2015). Many of the periods with the highest concentration of signal power align between the two series, suggesting that they capture the same solar physics. We note that the power concentrated at rotation and half-rotation periods in $\mathrm{RV}_{1}$ exceeds that of $\mathrm{RV}_{2}$, normalizing to the false alarm probability. While the rms scatter of $R V_{2}$ is greater than $R V_{1}$, we find that over $1.5 \mathrm{~m} \mathrm{~s}^{-1}$ of white noise would need to be added to $\mathrm{RV}_{2}$ compared to $\mathrm{RV}_{1}$ to account for this difference in peak heights. The model from M17 would predict, however, that since $\mathrm{RV}_{2}$ is calculated from lines presumably formed lower in the stellar photosphere, it should be more dominated by the suppression of convective blueshift, which would imply that this trend should be reversed. This observation presents the first suggestion that one of the assumptions that underlies the model is not realized on this data set.

\section{Analysis}

\subsection{Solving for $\alpha$}

If $\alpha$ is known, we can use experimentally determined values for $R V_{0}$ and $R V_{1}$ to extract theoretical time series of interest $R V_{\text {conv }}$ and $R V_{\text {sppl. }}$. This process should isolate contributions from the suppression of convective blueshift, and leave behind common-mode planetary and photometric contributions in the corrected RV time series. In practice, however, we must estimate the parameter $\alpha$ by imposing assumptions on the reconstructed time series. We adopt methods to solve for the parameter $\alpha$ based on assumptions made for the reconstructed time series from M17. These methods rely predominantly on the assumption that $\mathrm{RV}_{\text {conv }}$ dominates the $\mathrm{RV}$ time series. We applied the five methods detailed in M17 to solve numerically for the value of $\alpha$ that: (1) minimizes the mean absolute value

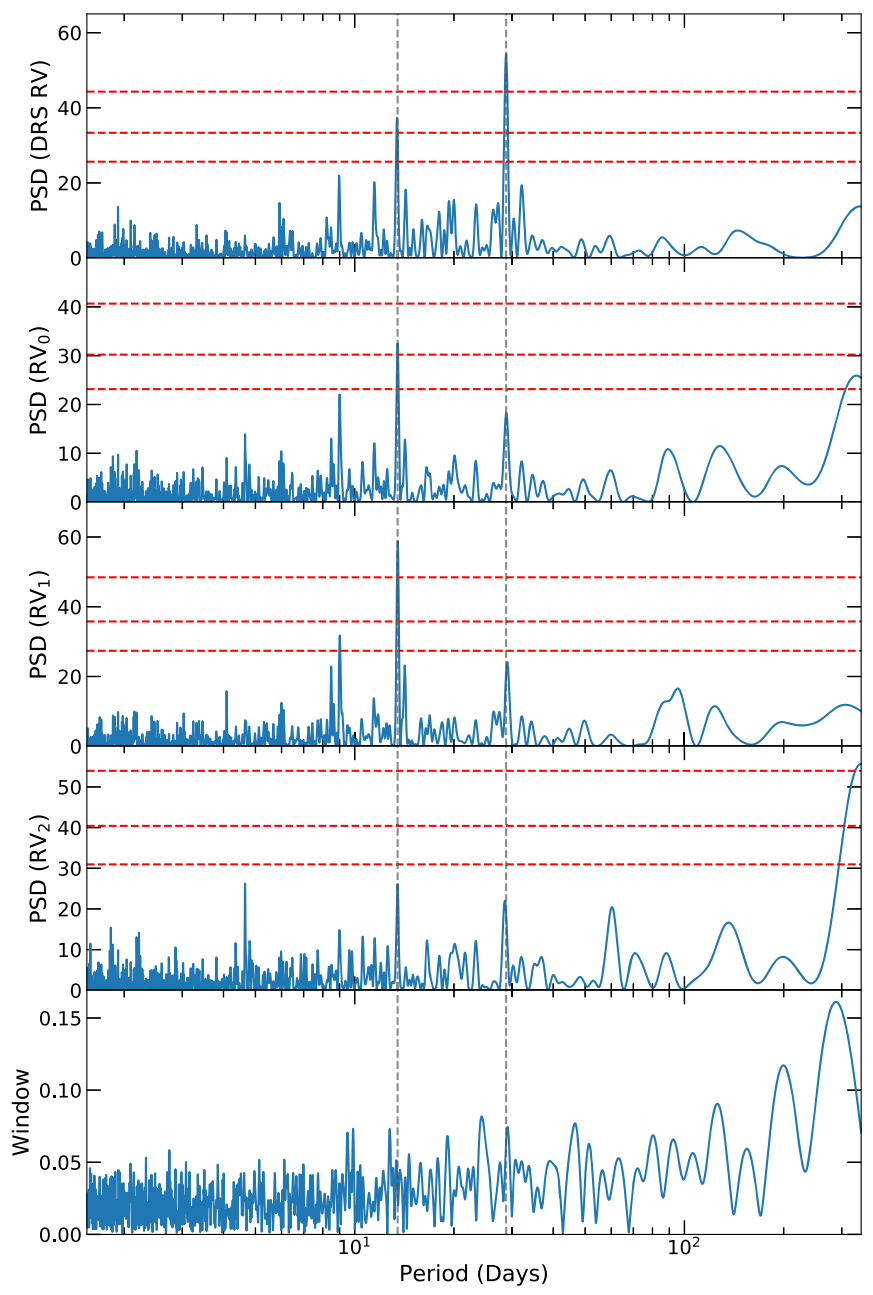

Figure 3. Lomb-Scargle periodograms of the DRS-reduced RVs, as well as the $\mathrm{RV}_{0}, \mathrm{RV}_{1}$, and $\mathrm{RV}_{2}$ time series generated from our line lists. The $10 \%, 1 \%$, and $0.1 \%$ false alarm probabilities (red dotted lines) are shown for each periodogram. The greatest power is concentrated in the solar synodic rotation period and its first harmonic (dotted gray lines), with no corresponding peaks in the window function (bottom panel).

of $\mathrm{RV}_{\text {sppl }}$; (2) minimizes the correlation between $\mathrm{RV}_{\text {conv }}$ and $\mathrm{RV}_{\text {sppl }}$; (3) is the slope of $\mathrm{RV}_{1}$ versus $\mathrm{RV}_{0}$; (4) maximizes the ratio of the variance in $\mathrm{RV}_{\text {conv }}$ versus that in $\mathrm{RV}_{\text {sppl }}$; (5) maximizes that ratio when $\mathrm{RV}_{\text {sppl }}$ is smoothed over 30 days, to average over rotationally modulated activity-induced variations. Additionally, (6) we calculate a best estimate for $\alpha$ as the ratio of the mean values of absolute $\mathrm{RV}$ time series derived from $\left\langle\mathrm{RV}_{1}\right\rangle /\left\langle\mathrm{RV}_{0}\right\rangle$ or $\left\langle\mathrm{RV}_{2}\right\rangle /\left\langle\mathrm{RV}_{0}\right\rangle$.

These values are shown in Table 4 . Despite the relatively consistent observational sampling and high $\mathrm{S} / \mathrm{N}$ of over 300 per exposure with an average of over 50 exposures per day, values for $\alpha$ differ based on the choice of assumption. Crucially, no physically motivated choice of $\alpha$ reduces the variability of $\mathrm{RV}_{\text {sppl }}$, the corrected $\mathrm{RV}$ time series, compared to $\mathrm{RV}_{0}$, the untreated time series, as illustrated in Figure 4.

\section{Discussion}

Despite the higher $\mathrm{S} / \mathrm{N}$ and better observational sampling for solar spectra, we are unable to extract physically significant reconstructed time series $\mathrm{RV}_{\text {conv }}$ and $\mathrm{RV}_{\text {sppl }}$ using the model and methods described in M17, suggesting that one of the assumptions of the model is not satisfied on this data set. 
Table 4

Estimates for $\alpha$, and rms Variation for Extracted $\mathrm{RV}_{\text {sppl }}$ Time Series $\left(\mathrm{m} \mathrm{s}^{-1}\right)$ from Different Methods

\begin{tabular}{|c|c|c|c|c|}
\hline Method & $\mathrm{RV}_{1}, \alpha$ & $\mathrm{RV}_{1}, \operatorname{std}\left(\mathrm{RV}_{\mathrm{sppl}}\right)$ & $\mathrm{RV}_{2}, \alpha$ & $\mathrm{RV}_{2}, \operatorname{std}\left(\mathrm{RV}_{\mathrm{sppl}}\right)$ \\
\hline (1) $\left\langle\mathrm{RV}_{\mathrm{sppl}}\right\rangle=0$ & .79 & 3.54 & 1.26 & 3.60 \\
\hline (2) Minimize correlation between $\mathrm{RV}_{\mathrm{sppl}}, \mathrm{RV}_{\text {conv }}$ & $\mathrm{N} / \mathrm{A}$ & $\mathrm{N} / \mathrm{A}$ & $\mathrm{N} / \mathrm{A}$ & $\mathrm{N} / \mathrm{A}$ \\
\hline (3) Slope of $R V_{i}$ versus $R V_{0}$ & .97 & 22.63 & 1.03 & 28.54 \\
\hline (4) Maximize $\operatorname{std}\left(\mathrm{RV}_{\text {conv }}\right) / \operatorname{std}\left(\mathrm{RV}_{\mathrm{sppl}}\right)$ & .99 & 67.79 & 1.01 & 85.56 \\
\hline (5) Maximize $\operatorname{std}\left(\mathrm{RV}_{\text {conv }}\right) / \mathrm{std}\left(\mathrm{RV}_{\mathrm{sppl}}\right.$ smoothed 30 days $)$ & .73 & 2.91 & 1.34 & 2.91 \\
\hline (6) $\left\langle\mathrm{RV}_{\mathrm{i}, \mathrm{abs}}\right\rangle /\left\langle\mathrm{RV}_{0, \mathrm{abs}}\right\rangle$ & .74 & 2.99 & 1.32 & 3.05 \\
\hline
\end{tabular}

Note. Method 2 fails to converge likely due to correlated noise, as discussed in M17.

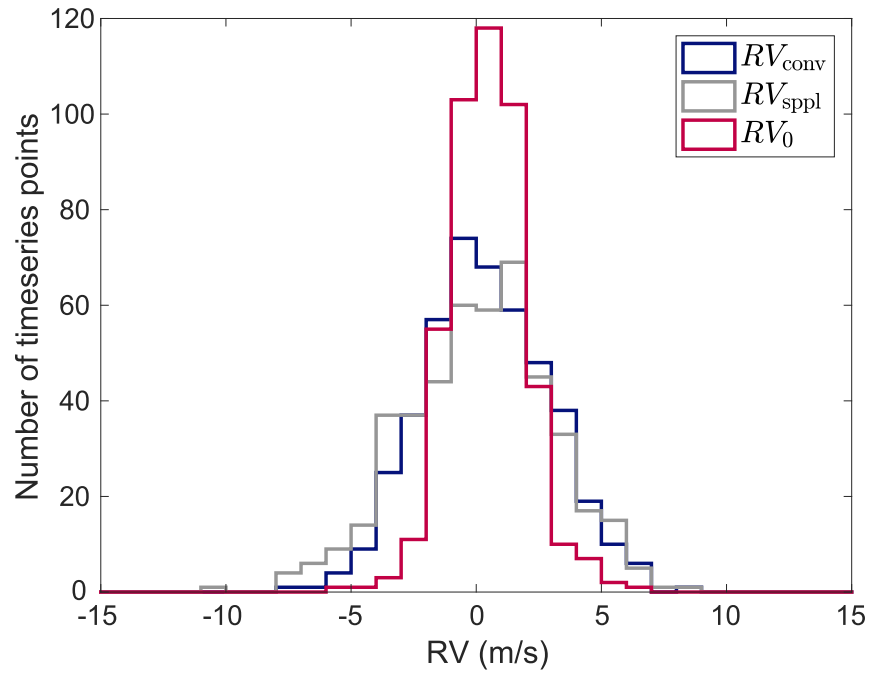

Figure 4. Histogram of time series points of $\mathrm{RV}_{0}$, and treated time series $\mathrm{RV}_{\text {sppl }}, \mathrm{RV}_{\text {sppl }}$ reconstructed using $\mathrm{RV}_{1}$ for $\alpha=.73$ : this reconstruction had the lowest $\mathrm{RV}$ rms for $\mathrm{RV}_{\mathrm{sppl}}$. As shown, this reconstruction fails to reduce the rms from the initial time series $\mathrm{RV}_{0}$.

Crucially, the methods to estimate $\alpha$ assume that $\mathrm{RV}_{\text {conv }}$ strongly dominates the RV time series. Potentially important is that our data set spans the activity minimum of the solar cycle, unlike the synthetic data set from M17, which included a full solar activity cycle: the assumption that $\mathrm{RV}_{\text {conv }}$ strongly dominates the RV time series might not hold for these restricted observations. Since the process of inverting the linear equations that describe $\left(\mathrm{RV}_{0}, \mathrm{RV}_{1}\right)$ to extract $\left(\mathrm{RV}_{\text {sppl }}, \mathrm{RV}_{\text {conv }}\right)$ amplifies all non- $\mathrm{RV}_{\text {conv }}$ contributions, weakened $\mathrm{RV}_{\text {conv }}$ at solar minimum could explain the inability to extract physical values of $\alpha$ on this data set. Our inability to reduce RV variability by applying the methods of M17 implies that sources of $\mathrm{RV}$ variability other than $\mathrm{RV}_{\text {conv }}$ must be taken into account.

Additional results in the literature have shown that other processes besides $\mathrm{RV}_{\text {conv }}$ may indeed play a dominant role near the solar minimum. For example, recent techniques demonstrate the ability to remove most power at the rotation period, but leave $1 \mathrm{~m} \mathrm{~s}^{-1} \mathrm{RV}$ variability in corrected time series. Dumusque (2018) and Cretingier et al. (2019) identify spectral lines insensitive to the suppression of convective blueshift; by computing RVs using these specially selected line lists, the authors are able to reduce the RV rms by a factor of 2.2, down to $0.9 \mathrm{~m} \mathrm{~s}^{-1}$. Independently, Milbourne et al. (2019) use solar images from $\mathrm{HMI} / \mathrm{SDO}$ to reproduce the activity-driven RVs. This analysis successfully removes the activity-driven signal at the rotation period, but still leaves an rms amplitude of 1.2 $\mathrm{m} \mathrm{s}^{-1}$. Using independent analysis frameworks, both techniques successfully remove the rotationally modulated activity signal, but are still limited by some other processes. Other work is ongoing to characterize the contributions from granulation and supergranulation, which can contribute as much as $1 \mathrm{~m} \mathrm{~s}^{-1}$ to RV rms (Dumusque et al. 2011; Meunier et al. 2015; Cegla et al. 2019; Meunier \& Lagrange 2019). The fact that our RV time series contain power concentrated at the rotation period and its harmonics is consistent with some significant $\mathrm{RV}_{\text {conv }}$ contribution. ${ }^{18}$ The inability to significantly reduce $\mathrm{RV}$ rms using the methods of M17 makes sense in the context of Milbourne et al. (2019) and Dumusque (2018), and the literature on granulation and supergranulation, which demonstrate that well over $1 \mathrm{~m} \mathrm{~s}^{-1}$ of $\mathrm{RV}$ variation remains after accounting for $\mathrm{RV}_{\text {conv }}$.

When the linear equations defining $R V_{0}$ and $R V_{1}$ in terms of $R V_{\text {sppl }}$ and $R_{\text {conv }}$ are inverted in the presence of noise introduced by this external variability, the rms of noise in $\mathrm{RV}_{\mathrm{sppl}}$ is magnified to twice as large as the original rms of noise in $\mathrm{RV}_{0}(\mathrm{M} 17)$. Potential contributions from instrumental systematics due to wavelength calibration (Cosentino et al. 2014; Dumusque 2018; Cersullo et al. 2019; Coffinet et al. 2019), or daily calibration sequences (Collier Cameron et al. 2019), may also contribute significantly to this non- $\mathrm{RV}_{\text {conv }} \mathrm{RV}$ variability. This sensitivity to RV contributions other than $\mathrm{RV}_{\text {conv }}$ motivates future consideration of different solar activity processes, especially those operating on different timescales such as magnetoconvection (Palle et al. 1995; Del Moro 2004; Meunier 2018). Furthermore, these additional processes, and even the suppression of convective blueshift itself, may contain subtle line list dependency, based on proxies for line responsiveness to magnetic activity such as the Lande-g factor (e.g., Norton et al. 2006). All of these contributions must be accounted for in order to reach the $10 \mathrm{~cm} \mathrm{~s}^{-1}$ detection limit of an Earth-like planet orbiting a Sun-like star. Future work is needed to identify correlations in spectra, solar images, or some other ancillary data set that could be used to model these phenomena.

This work was supported in part by NASA award number NNX16AD42G and the Smithsonian Institution. This work is based on observations made with the Italian Telescopio Nazionale Galileo (TNG) operated by the Fundación Galileo Galilei (FGG) of the Istituto Nazionale di Astrofisica (INAF) at the Observatorio del Roque de los Muchachos (La Palma,

\footnotetext{
18 We note, however, that the concentration of high-activity regions separated by $180^{\circ}$ longitude on the Sun containing not only plage but also long-lived sunspots that contribute to $\mathrm{RV}_{\text {sppl }}$ through the photometric effect can also supply power at the rotation period (Schroeter 1984; Shelke \& Verma 1985); similar structure exists on other Sun-like stars (Berdyugina \& Järvinen 2005).
} 
Canary Islands, Spain). The solar telescope used in these observations was built and maintained with support from the Smithsonian Astrophysical Observatory, the Harvard Origins of Life Initiative, and the TNG.

This work was performed in part under contract with the California Institute of Technology (Caltech)/Jet Propulsion Laboratory (JPL) funded by NASA through the Sagan Fellowship Program executed by the NASA Exoplanet Science Institute (R.D.H.).

The authors thank A. Ravi for his assistance in preparing and submitting this manuscript.

A.C.C. acknowledges support from STFC consolidated grant number ST/M001296/1.

D.W.L. acknowledges partial support from the Kepler mission under NASA Cooperative Agreement NNX13AB58A with the Smithsonian Astrophysical Observatory.

S.S. acknowledges support by NASA Heliophysics LWS grant NNX16AB79G.

H.M.C. acknowledges the financial support of the National Centre for Competence in Research PlanetS supported by the Swiss National Science Foundation (SNSF)

X.D. is grateful to the Branco-Weiss fellowship-Society in Science for continuous support.

This publication was made possible through the support of a grant from the John Templeton Foundation. The opinions expressed are those of the authors and do not necessarily reflect the views of the John Templeton Foundation.

This material is based upon work supported by the National Aeronautics and Space Administration under grants No. NNX15AC90G and NNX17AB59G issued through the Exoplanets Research Program. The research leading to these results has received funding from the European Union Seventh Framework Programme (FP7/2007-2013) under grant Agreement No. 313014 (ETAEARTH).

This work was supported in part by the NSF-REU solar physics program at SAO, grant No. AGS-1560313.

The HARPS-N project has been funded by the Prodex Program of the Swiss Space Office (SSO), the Harvard University Origins of Life Initiative (HUOLI), the Scottish Universities Physics Alliance (SUPA), the University of Geneva, the Smithsonian Astrophysical Observatory (SAO), and the Italian National Astrophysical Institute (INAF), the University of St Andrews, Queen's University Belfast, and the University of Edinburgh.

This research has made use of NASA's Astrophysics Data System.

We thank the entire TNG staff for their continued support of the solar telescope project at HARPS-N.

Facility: TNG:HARPS-N.

\section{ORCID iDs}

T. W. Milbourne (i) https://orcid.org/0000-0001-5446-7712

R. D. Haywood (1) https://orcid.org/0000-0001-9140-3574

H. M. Cegla (1) https://orcid.org/0000-0001-8934-7315

X. Dumusque (1) https://orcid.org/0000-0002-9332-2011

J. Maldonado (1) https://orcid.org/0000-0002-2218-5689

L. Malavolta (ib https://orcid.org/0000-0002-6492-2085

A. Mortier (1) https://orcid.org/0000-0001-7254-4363

A. Ghedina (1) https://orcid.org/0000-0003-4702-5152

M. López-Morales (1) https://orcid.org/0000-0003-3204-8183

E. Molinari (i) https://orcid.org/0000-0002-1742-7735

Ennio Poretti (i) https://orcid.org/0000-0003-1200-0473
D. Sasselov (1) https://orcid.org/0000-0001-7014-1771

A. Sozzetti (i) https://orcid.org/0000-0002-7504-365X

\section{References}

Baranne, A., Queloz, D., Mayor, M., et al. 1996, A\&AS, 119, 373

Berdyugina, S. V., \& Järvinen, S. P. 2005, AN, 326, 283

Bergemann, M., Gallagher, A. J., Eitner, P., et al. 2019, A\&A, 631, A80

Borucki, W. J., Koch, D., Basri, G., et al. 2010, Sci, 327, 977

Cegla, H. M., Shelyag, S., Watson, C. A., \& Mathioudakis, M. 2013, ApJ, 763, 95

Cegla, H. M., Watson, C. A., Shelyag, S., Mathioudakis, M., \& Moutari, S. 2019, ApJ, 879, 55

Cersullo, F., Coffinet, A., Chazelas, B., Lovis, C., \& Pepe, F. 2019, A\&A, 624, A122

Coffinet, A., Lovis, C., Dumusque, X., \& Pepe, F. 2019, A\&A, 629, A27

Collier Cameron, A., Mortier, A., Phillips, D., et al. 2019, MNRAS, 487, 1082

Cosentino, R., Lovis, C., Pepe, F., et al. 2012, Proc. SPIE, 8446, 84461V

Cosentino, R., Lovis, C., Pepe, F., et al. 2014, Proc. SPIE, 9147, 91478C

Cretingier, M., Dumusque, X., Allart, R., Pepe, F., \& Lovis, C. 2019, A\&A, submitted

Del Moro, D. 2004, A\&A, 428, 1007

Dravins, D., Lindegren, L., \& Nordlund, A. 1981, A\&A, 96, 345

Dumusque, X. 2018, A\&A, 620, A47

Dumusque, X., Boisse, I., \& Santos, N. C. 2014, ApJ, 796, 132

Dumusque, X., Glenday, A., Phillips, D. F., et al. 2015, ApJL, 814, L21

Dumusque, X., Udry, S., Lovis, C., Santos, N. C., \& Monteiro, M. J. P. F. G. 2011, A\&A, 525, A140

Fischer, D. A., Anglada-Escude, G., Arriagada, P., et al. 2016, PASP, 128 , 066001

Giorgini, J. D., Yeomans, D. K., Chamberlin, A. B., et al. 1996, BAAS, 28, 1158

Gray, D. 2005, The Observation and Analysis of Stellar Photospheres (Cambridge: Cambridge Univ. Press)

Gray, D. F. 2009, ApJ, 697, 1032

Gray, D. F., \& Oostra, B. 2018, ApJ, 852, 42

Haywood, R. D., Collier Cameron, A., Unruh, Y. C., et al. 2016, MNRAS, 457,3637

Howell, S. B., Sobeck, C., Haas, M., et al. 2014, PASP, 126, 398

Isaacson, H., \& Fischer, D. 2010, ApJ, 725, 875

Kurucz, R. L., Furenlid, I., Brault, J., \& Testerman, L. 1984, Solar Flux Atlas from 296 to $1300 \mathrm{~nm}$ (Sunspot, NM: National Solar Observatory)

Mayor, M., \& Queloz, D. 1995, Natur, 378, 335

Meunier, N. 2018, A\&A, 615, A87

Meunier, N., Desort, M., \& Lagrange, A.-M. 2010a, A\&A, 512, A39

Meunier, N., \& Lagrange, A.-M. 2019, A\&A, 625, L6

Meunier, N., Lagrange, A.-M., \& Borgniet, S. 2017a, A\&A, 607, A6

Meunier, N., Lagrange, A.-M., Borgniet, S., \& Rieutord, M. 2015, A\&A, 583, A118

Meunier, N., Lagrange, A.-M., \& Desort, M. 2010b, A\&A, 519, A66

Meunier, N., Lagrange, A.-M., Mbemba Kabuiku, L., et al. 2017b, A\&A, 597, A52

Milbourne, T. W., Haywood, R. D., Phillips, D. F., et al. 2019, ApJ, 874, 107

Motalebi, F., Udry, S., Gillon, M., et al. 2015, A\&A, 584, A72

Nordlund, A., Stein, R. F., \& Asplund, M. 2009, LRSP, 6, 2

Norton, A. A., Graham, J. P., Ulrich, R. K., et al. 2006, SoPh, 239, 69

Palle, P. L., Jimenez, A., Perez Hernandez, F., et al. 1995, ApJ, 441, 952

Pepe, F. A., Cristiani, S., Lopez, R. R., et al. 2010, Proc. SPIE, 7735, 77350F

Phillips, D. F., Glenday, A. G., Dumusque, X., et al. 2016, Proc. SPIE, 9912, 99126Z

Reiners, A., Mrotzek, N., Lemke, U., Hinrichs, J., \& Reinsch, K. 2016, A\&A, 587, A65

Ricker, G. R., Winn, J. N., Vanderspek, R., et al. 2014, JATIS, 1, 014003

Saar, S. H., \& Donahue, R. A. 1997, ApJ, 485, 319

Schrijver, C. J., \& Zwaan, C. 2000, Solar and Stellar Magnetic Activity (Cambridge: Cambridge Univ. Press)

Schroeter, E. H. 1984, A\&A, 139, 538

Shelke, R. N., \& Verma, V. K. 1985, BASI, 13, 53

Sosnowska, D., Lodi, M., Gao, X., et al. 2012, Proc. SPIE, 8451, 84511X

Stein, R. F. 2012, LRSP, 9, 4

VanderPlas, J., Connolly, A. J., Ivezić, Ž., \& Gray, A. 2012, in IEEE Conf. on Intelligent Data Understanding (Piscataway, NJ: IEEE), 47

VanderPlas, J. T., \& Ivezić, Ž. 2015, ApJ, 812, 18

Zechmeister, M., \& Kürster, M. 2009, A\&A, 496, 577 